\title{
Novel Function of GTP-Binding Protein and Ribosome in Initiating Cell Differentiation in Streptomyces
}

\author{
Susumu Okamoto \\ National Food Research Institute, 2-1-12 Kannondai, Tsukuba, Ibaraki 305-8642, Japan \\ E-mail: sdspage@affrc.go.jp \\ (Received Dec. 4, 2001)
}

\section{INTRODUCTION}

During evolution organisms have learned to respond to certain environmental changes by producing specialized cell types. Several genera of bacteria including Bacillus, Clostridium, Sporosarcina, Myxococcus and Streptomyces produce spores when they encounter adverse environmental conditions such as nutrient deficiency. A distinguishing characteristic of species of the genus Streptomyces is their ability to form an aerial mycelium from a substrate mycelium when cultured on solid media, eventually leading to the formation of spores by the synchronous and regularly spaced septation of the aerial mycelium (morphological differentiation). In addition to this characteristic feature, streptomycetes are of interest because they produce many antibiotics (physiological differentiation) with important uses in medical and agricultural fields. Most of these antibiotics are synthesized by complex biosynthetic pathways and expressions of these biosynthetic genes occur during the onset of morphological differentiation in agar plate cultures. Although much effort has been made, the mechanisms by which morphological and/or physiological differentiation are regulated remain to be clarified. In this paper, I describe the molecular analysis of the GTP-binding protein "Obg" which regulates morphological differentiation and discovery of novel function of ribosomes which modulates antibiotic production in Streptomyces.

\section{Functional Analysis of $o b g$ Gene}

Our laboratory ${ }^{1-3)}$ and the laboratory of Freese ${ }^{4-6)}$ have demonstrated that a decrease in GTP pool size correlates with the initiation of morphological differentiation in Bacillus subtilis and Streptomyces spp.. Thus, these organisms can be induced to differentiate in nutritionally rich media, in which cells normally do not sporulate: i) when the GTP pool size is reduced by the addition of decoyinine, an inhibitor of GMP synthesis; ii) by the depletion of guanine in guanine-requiring auxotrophic mutants; or iii) by provoking the stringent response in which ppGpp, a potent inhibitor of IMP-dehydrogenase, accumulates. Although the role of GTP pool size variations is at present unclear, it seems logical that a GTP-binding protein(s) could be involved in sensing a decreased GTP level as a signal for differentiation, analogous to the GTP-binding protein mediated signal transduction systems in eucaryotes such as the Ras signaling pathway.

The Obg protein of Bacillus subtilis, one of the well characterized procaryoic GTP-binding proteins, is encoded by the $o b g$ gene, which is just downstream of the stage 0 sporulation gene $s p o O B^{7}$. Although the detailed function of Obg has not been delineated, it was shown that Obg could play a role in regulating the initiation of sporulation in this organism ${ }^{8-10}$ ). In order to confirm the existence of the obg homolog and its possible involvement in differentiation in Streptomyces spp, we attempted to clone the $o b g$ gene from S. griseus IFO13189 and S. coelicolor A3(2) ${ }^{11)}$.

obg homolog of S. griseus was cloned by PCR using degenerate oligonucleotide primers which are designed based on the conserved amino acid sequences found within the $\mathrm{Obg} / \mathrm{Gtp} 1 \mathrm{GTPase}$ family. The $S$. griseus obg gene was located just downstream of the genes for ribosomal proteins L21 and L27, encoded a protein of 478 amino acids $(51 \mathrm{kDa})$, and possessed three consensus GTPbinding motifs that are designated G1 (consensus: G/AXXXXGKT/S), G3 (consensus: DXXG), and G4 (consensus: NKXD). The Obg protein expressed in E. coli bound GTP, as demonstrated using a UV cross-linking method. Western blot analysis revealed that Obg protein level sharply decreases just after the onset of aerial mycelium formation or at the end of vegetative growth on agar palate cultures, indicating that expression of $o b g$ is regulated in a growth phase-dependent manner. Introduction of multiple copies of obg into wild-type $S$. griseus suppressed aerial mycelium development in solid culture and submerged spore formation in liquid culture (Fig. 1). No effect on streptomycin production was detected, indicating that Obg is involved in the regulation of the onset of morphological but not physiological differentiation in this organism.

The S. coelicolor obg gene was identified by Southern hybridization using $S$. griseus obg gene as a probe ${ }^{11}$. The $S$. coelicolor obg gene located between $p r o A$ and $\operatorname{clp} P, X$ on its chromosome (on cosmid C88) and encoded a protein of 478 amino acids (51 kDa) with $85.7 \%$ sequence identity to the $S$. griseus Obg protein ${ }^{12)}$. Gene disruption experiment indicated that the $o b g$ gene product was essential for growth. Propagation of $o b g$ gene with multi-copy plasmid suppressed aerial mycelium formation of $S$. coelicolor as was the case of S. griseus (Fig. 2B). To clarify whether the Obg protein regulates cell differentiation through its ability to bind GTP, site-directed mutagenesis techniques were employed to introduce mutations within the consensus regions (G1 and G4) and the resulting mutant genes were propagated in wild-type cells using multi-copy plasmid 


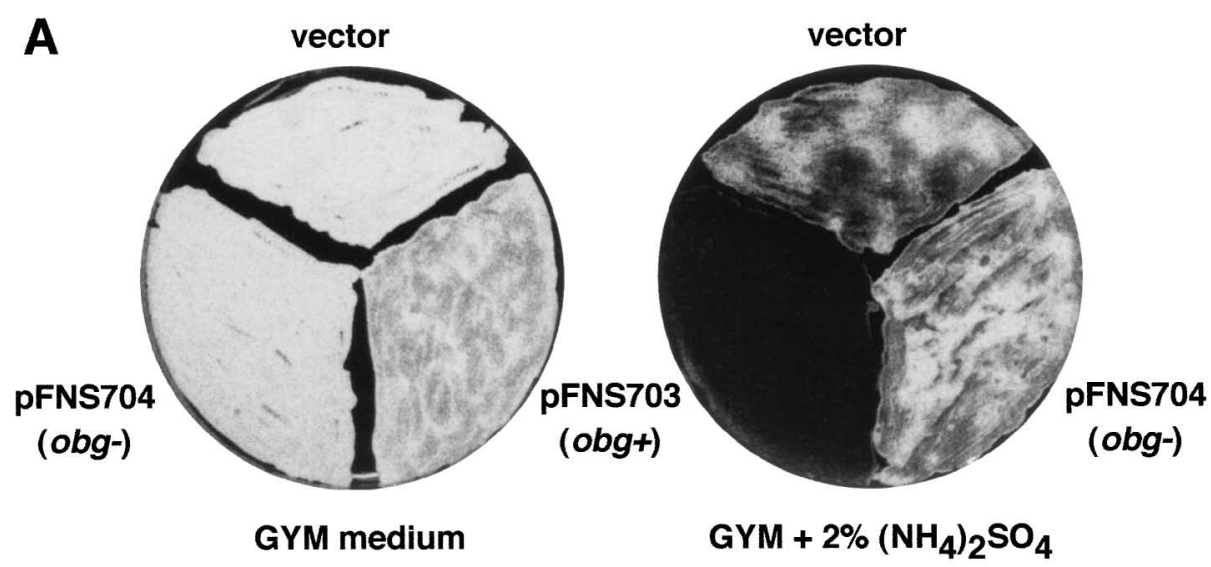

B
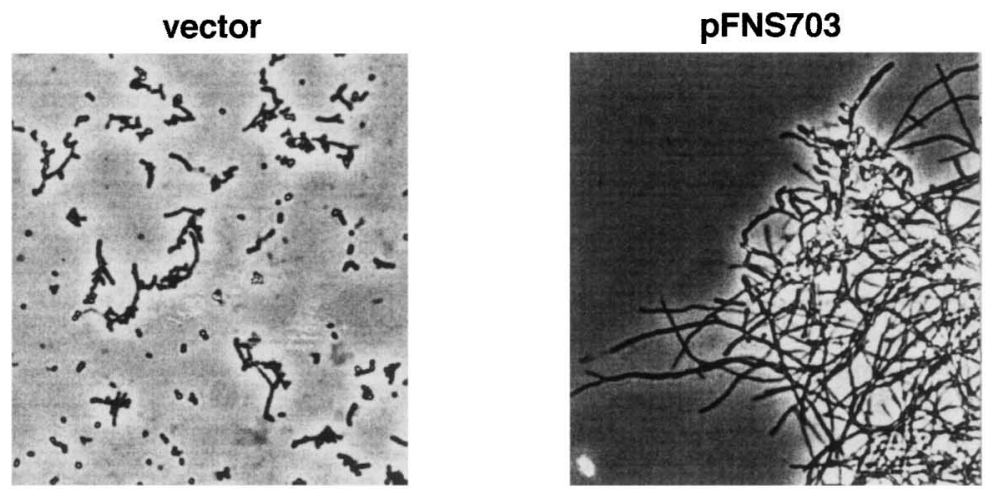

Fig. 1. Effect of multicopy obg on aerial mycelium formation (A) or submerged spore formation (B) in Streptomyces griseus IFO13189. The plasmid pFNS704 contains the nonfunctional obg gene which has a frameshift mutation near its N-terminal end.

Table 1. Streptomycin resistant mutations in S. coelicolor and S. lividans.

\begin{tabular}{cccc}
\hline Strain & $\begin{array}{c}\text { Mutation in } \\
r p s L \text { gene }^{\mathrm{a}}\end{array}$ & $\begin{array}{c}\text { Resistance to } \\
\text { streptomycin } \\
(\mu \mathrm{g} / \mathrm{ml})\end{array}$ & $\begin{array}{c}\text { Actinorhodin } \\
\text { productivity }\end{array}$ \\
& &
\end{tabular}

\section{S. lividans}

TK21

TK24 (str-6)

$\stackrel{-}{\text { Lys } 88 \rightarrow \text { Glu }}$

1
100

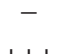

S. coelicolor

\begin{tabular}{lcrc}
1147 (wild-type) & - & 1 & + \\
KO-192 (strA1) & Lys43 $\rightarrow$ Asn & 200 & + \\
KO-178 (str-18) & Lys88 $\rightarrow$ Glu & 100 & +++ \\
KO-179 (str-19) & None (ND) & 5 & +++ \\
& - & 1 & - \\
M570 (relA) & - & 50 & + \\
KO-140 (relA str) & Lys88 $\rightarrow$ Arg & & - \\
\#27 (brgA) & - & 1 & ++ \\
KO-150 (brgA str $)$ & Arg86 $\rightarrow$ His & 5 & + \\
\hline
\end{tabular}

a -; wild-type $r p s L$
(Fig. 2A). Strikingly, propagation of one of the mutant $o b g$ gene (P168V) exerted more accentuated suppressive effect on aerial mycelium formation than did the wild-type $o b g$ gene propagation (Fig. 2B). In contrast, we found a surprising fact that propagation of another mutant obg gene (G171A) results in an enhancement of aerial mycelium formation (i.e. gain of function mutation). Moreover, this transformant revealed an increased actinorhodin production. No such phenotypic changes were detected by introduction of other four mutant obg genes (K172N, S173N, N282I, and D285A).

According to these results, we proposed that the Obg protein plays a crucial role in sensing changes in the nutritional environment, eventually leading to cell differentiation.

\section{Ribosome Function Modulates Antibiotic Production}

One of the well-studied regulatory systems for antibiotic production in bacteria is the stringent response, which is initiated by nutrient limitation. The highly phosphorylated guanine nucleotides ppGpp and pppGpp have been shown to be responsible for the stringent response ${ }^{13)}$. The synthesis of (p)ppGpp is catalyzed by ribosome-bound RelA pro- 

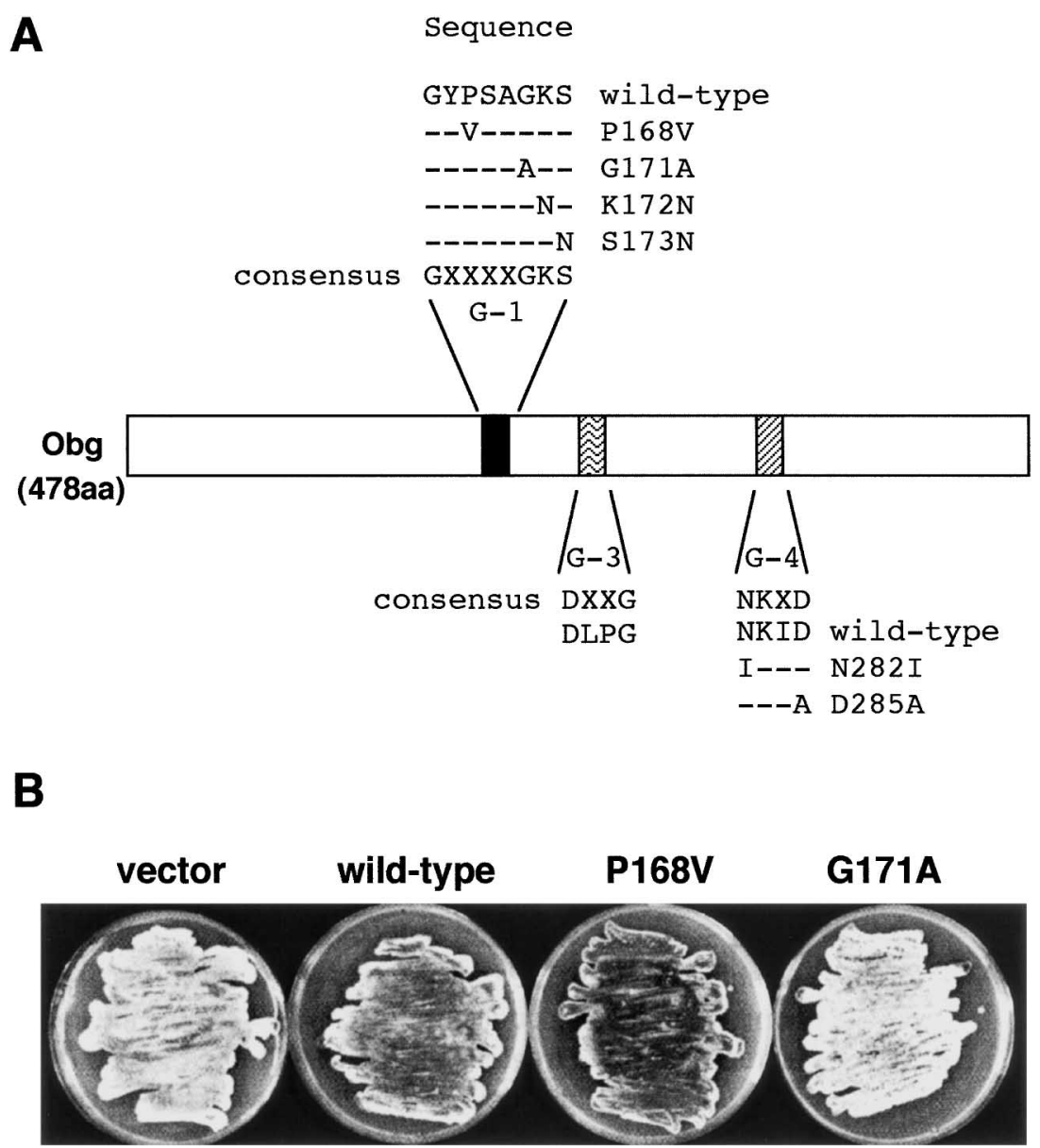

Fig. 2. Site-directed mutagenesis of the GTP-binding domain of the Obg protein (A) and effect of overproduction of the constructed mutant Obg proteins on morphological differentiation in Streptomyces coelicolor (B).

tein, which is activated when uncharged tRNA enters the A site on a ribosome. The $50 \mathrm{~S}$ ribosomal protein L11 encoded by the relC gene $(=r p l K)$ is also required for activation of the ribosome-bound RelA protein. Initiation of antibiotic production in streptomycetes should require (p)ppGpp. Thus, mutations in relA or relC gene result in loss of antibiotic productivity ${ }^{3,14)}$.

Such advances in understanding the regulation of antibiotic production in genus Streptomyces have come from the study of antibiotic synthesis in Streptomyces coelicolor A3(2) and Streptomyces lividans 66. S. coelicolor produces at least four antibitics [actinorhodin (Act), undecylprodigiosin (Red), calicum-dependent antibiotic (CDA), and methylenomycin (Mmy)]. Because Act and Red are blue- and red-pigmented antibiotics respectively, production of these antibiotics is easily detected by eye-inspection. This fact makes this strain particularly appropriate for studying the regulation of antibiotic production. The closely related strain $S$. lividans (including strain TK21) normally does not produce antibiotics (Act and Red), although the strain has complete sets of biosynthetic genes for these antibiotics.
Several years ago, we have found that a strain of S. lividans, TK24, produces Act, and a streptomycin resistant mutation (str-6) in this strain is responsible for induction of Act synthesis (Fig. 3) ${ }^{15}$. From the further analyses, it was also revealed that introduction of streptomycin resistant mutations improves actinorhodin production in wildtype $S$. coelicolor, and circumvents the detrimental effects on antibiotic production in certain developmental mutants (relA, relC and $\operatorname{brgA}$ ) of $S$. coelicolor ${ }^{15,16)}$. These mutations result in the alteration of the Lys- 88 amino acid in ribosomal protein $\mathrm{S} 12$ (encoded by $r p s L$ gene) to Glu or Arg (Table 1). In addition to the rpsL mutations, other mutations conferring low level resistance to streptomycin also exhibited a similar increase in Act production, but no mutation in the rpsL gene was detected (ND mutation). These findings indicate that the antibiotic production in streptomycetes is significantly controlled by the translational machinery "ribosome".

Expression of Act biosynthetic genes has been shown to depend on a gene designated actII-ORF4 ${ }^{17}$. Similarly, redZ and redD are required for expression of Red synthetic genes ${ }^{18,19)}$. ActII-ORF4, RedZ and RedD are transcription- 
al activators (DNA binding protein) and have been termed "pathway-specific activators". The expression level of these pathway-specific activators directly determines the production level of each antibiotic. First, we confirmed whether activation of Act production by $r p s L$ mutations requires the ActII-ORF4 protein. We constructed actIIORF4- mutants from S. lividans TK24 and S. coelicolor KO-178 (Both strains harbor rpsL-K88E mutation). The constructed rpsL actII-ORF4- double mutants completely impaired in Act production, indicating that the rpsL mutation could not by-pass the function of ActII-ORF4 protein (Fig. 3). Next, we attempted to assess the expression level of ActII-ORF4 protein (Fig. 4). For this purpose, we pre-

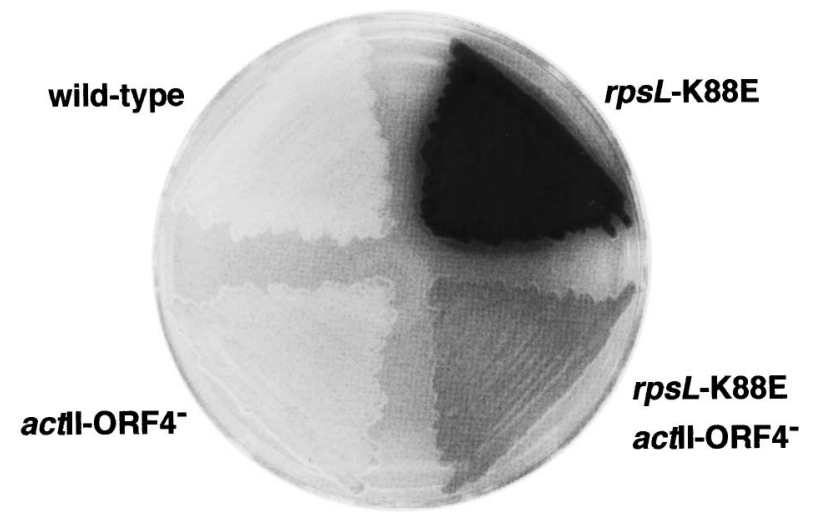

Fig. 3. Effect of actII-ORF4 disruption on actinorhodin production in the rpsL-K88E mutant of S. lividans. pare the antibodies against ActII-ORF4 proteins and used in Western blot analysis. The results showed that expression of ActII-ORF4 protein strongly enhanced by rpsLK88E mutation. Furthermore, RT-PCR experiment revealed that the increase of ActII-ORF4 level is attributed to the enhanced expression of actII-ORF4 mRNA.

Thus, rpsL-K88E mutation enhances expression of actII-ORF4 gene, leading to massive production of Act. However, the mechanism by which the rpsL mutations activate the expression of actII-ORF4 gene is still remained to be clarified.

\section{ACKNOWLEDGEMENTS}

I would like to express my sincere gratitude to Prof. Tatsuji Seki, the chairman of the Society for Actinomycetes Japan (SAJ), the members of the award nomination committee and the board of directors of SAJ for honoring me with the Hamada Award (SAJ Encouragement Award). I am most grateful to Dr. Ochi and all of collaborators in National Food Research Institute for encouragement, advice and great collaboration. I express my thanks to Prof. Yasuhiro Yamada (Fukuyama University), Associate Prof. Takuya Nihira (Osaka University) and Associate Prof. Shohei Sakuda (University of Tokyo) for encouragement.

A

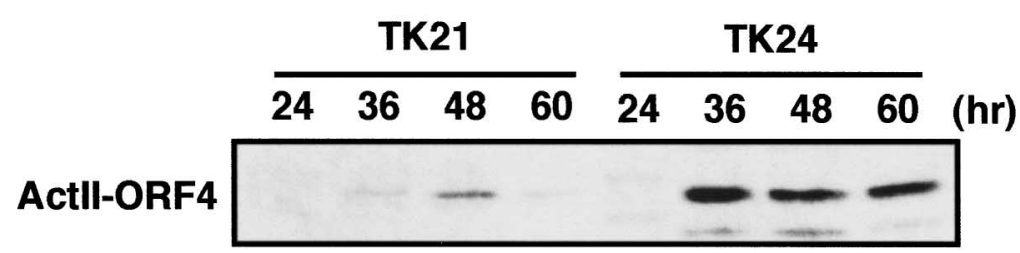

B
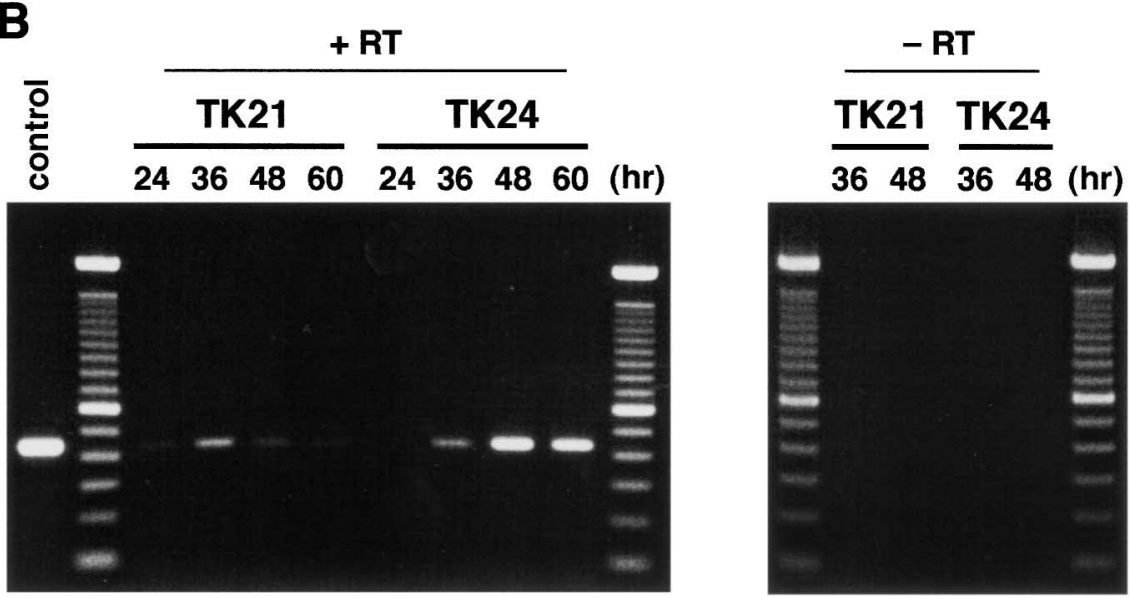

Fig. 4. Western blot analysis of ActII-ORF4 protein (A) and determination of actII-ORF4 mRNA level by RT-PCR (B). 


\section{REFERENCES}

1) Ochi, K.: Metabolic initiation of differentiation and secondary metabolism by Streptomyces griseus: significance of the stringent response (ppGpp) and GTP content in relation to A-factor. J. Bacteriol. 169: 3608-3616, 1987.

2) Ochi, K.: Changes in nucleotide pools during sporulation of Streptomyces griseus in submerged culture. J. Gen. Microbiol. 133: 2787-2795, 1987.

3) Ochi, K.; D. Zhang, S. Kawamoto \& A. Hesketh: Molecular and functional analysis of the ribosomal L11 and S12 protein genes (rplK and rpsL) of Streptomyces coelicolor A3(2) Mol. Gen. Genet. 256: 488-498, 1997.

4) Freese, E. \& J. Heinze: Metabolic and genetic control of bacterial sporulation. In "The bacterial spores, vol. 2" (Eds. A. Hurst, G. W. Gould \& J. Dring), pp 101-172, Academic Press, London, UK, 1984.

5) Lopez, J. M.; A. Dromerick \& E. Freese: Response of guanosine-5' -triphosphate concentration to nutritional changes and its significance for Bacillus subtilis sporulation. J. Bacteriol. 146: 605-613, 1981.

6) Ochi, K.; J. C. Kandala \& E. Freese: Initiation of Bacillus subtilis sporulation by the stringent response to partial amino acid deprivation. J. Biol. Chem. 256: 6866-6875, 1981.

7) Trach, K. \& J. A. Hoch: The Bacillus subtilis spo0B stage 0 sporulation operon encodes an essential GTP-binding protein. J. Bacteriol. 171: 1362-1371, 1989.

8) Kok, J.; Trach, K. A. \& J. A. Hoch: Effects on Bacillus subtilis of a conditional lethal mutation in the essential GTPbinding protein Obg. J. Bacteriol. 176: 7155-7160, 1994.

9) Welsh, K. M.; Trach K. A.; Folger, C. \& J. A. Hoch: Biochemical characterization of the essential GTP-binding protein Obg of Bacillus subtilis. J. Bacteriol. 176: 7161$7168,1994$.

10) Vidwans, S. J.; Ireton, K. \& A. D. Grossman: Possible role for the essential GTP-binding protein Obg in regulating the initiation of sporulation in Bacillus subtilis. J. Bacteriol. 177: 3308-3311, 1995.

11) Okamoto, S.; M. Itoh \& K. Ochi: Molecular cloning and characterization of the obg gene of Streptomyces griseus in relation to the onset of morphological differentiation. J. Bacteriol. 179: 170-179, 1997.

12) Okamoto, S \& K. Ochi: An essential GTP-binding protein functions as a regulator for differentiation in Streptomyces coelicolor. Mol. Microbiol. 30, 107-119, 1998.

13) Cashel, M.; Gentry, D. R.; Hernandez, V. J. \& D. Vinella: The stringent response. In "Esherichia coli and Salmonoella typhimurium: cellular and molecular biology" (Eds. F. C. Neidhardt, R. Curtiss, J. L. Ingraham, E. C. C. Lin, K. B. Low, B. Magasanik, W. S. Reznikoff, M. Riley, M. Schaechter \& H. E. Umbarger), pp 1410-1438, American Society for Microbiology, Washington, D.C., 1996.

14) Chakraburtty, R. \& M. Bibb: The ppGpp synthetase gene (relA) of Streptomyces coelicolor A3(2) plays a conditional role in antibiotic production and morphological differentiation. J. Bacteriol. 179: 5854-5861, 1997.

15) Shima, J.; Hesketh, A. Okamoto, S., Kawamoto, S. \& K. Ochi: Induction of actinorhodin production by $r p s L$ (encoding ribosomal protein S12) mutations that confer streptomycin resistance in Streptomyces lividans and Streptomyces coelicolor A3(2). J. Bacteriol. 178: 7276-7284, 1996.

16) Hesketh, A. \& K. Ochi: A novel method for improving Streptomyces coelicolor A3(2) for production of actinorhodin by introduction of $r p s L$ (encoding ribosomal protein S12) mutations conferring resistance to streptomycin. J. Antibiot. 50: 532-535, 1997.

17) Gramajo, H. C.; Takano, E. \& M. J. Bibb: Stationary-phase production of the antibiotic actinorhodin in Streptomyces coelicolor A3(2) is transcriptionally regulated. Mol. Microbiol. 7: 837-845, 1993.

18) Takano, E.; Gramajo, H. C.; Strauch, E., Andres, N., White, J. \& M. J. Bibb: Transcriptional regulation of the redD transcriptional activator gene accounts for growth-phase dependent production of the antibiotic undecylprodigiosin in Streptomyces coelicolor A3(2). Mol. Microbiol. 6: 27972804, 1992.

19) White, J. \& M. J. Bibb: bldA dependence of undecylprodigiosin production in Streptomyces coelicolor A3(2) involves a pathway-specific regulatory cascade. J. Bacteriol. 179: 627-633, 1997. 